\title{
Association between family structure and food group intake in children
}

\author{
Youn Joo Baek', Hee Young Paik ${ }^{1}$ and Jae Eun Shim ${ }^{2 \S}$ \\ 'Department of Food and Nutrition, Seoul National University, Seoul 151-742, Korea \\ ${ }^{2}$ Department of Food and Nutrition, Daejeon University, 62 Daehak-ro, Dong-gu, Daejeon 300-716, Korea
}

BACKGROUNDS/OBJECTOVES: Family has an impact on dietary intake of children as a proximal food environment and family structures are changing and becoming more diverse. This study was performed to identify the association between family structure and food group intake of children aged 3-18 years in Korea.

SUBJECTS/METHODS: A total of 3,217 children with complete data on variables for household information, dietary intake and sampling weights were obtained from 2010-2011 Korea National Health and Nutrition Examination Survey. Children's family structure was classified into 3 groups (Living with both parents, Living with one parent and Living without parents). To evaluate children's food group intake, scores of individual food groups ('Grains', 'Meat, Fish, Eggs and Beans', 'Vegetables', 'Fruits' and 'Milk and Dairy products') was calculated from percent adherence to the recommended servings of the Korean Food Guidance System (KFGS). 'Food group mean score' was obtained by calculating the average of five food group scores.

RESULTS: After adjusting for age, sex, number of family members, and household income, children living with both parents had higher scores in 'Fruits' $(P<0.01)$, 'Milk and Dairy products' $(P<0.05)$, and mean score of individual food group score $(P<0.001)$ compared to children living with one parent. Individual food group scores and mean scores of individual food group scores were associated with different socio-demographic factors in study children. Family structure was associated with 'Fruits,' 'Milk and Dairy products' score and mean scores of food group scores.

CONCLUSIONS: These results suggested that different approach might be required to solve nutrition problem in children depending on their family structure and other socio-demographic factors.

Nutrition Research and Practice 2014;8(4):463-468; doi:10.4162/nrp.2014.8.4.463; pISSN 1976-1457 elSSN 2005-6168

Keywords: Family structure, children, food group intake, Korean food guidance system

\section{INTRODUCTION}

Children continue to grow and develop physically, cognitively, emotionally, and socially as they transit into adulthood [1]. Adequate nutrition status and healthy eating habit during this period are fundamental to children's growth and development, and ultimately protect them from chronic diseases (e.g. heart disease, cancer, and diabetes etc.) later in their life [2]. Furthermore, obese child had 2-6.5 folds higher risk for becoming an obese adult compared to non-obese child [3]. Therefore healthful dietary intake during this period carries an important meaning not just in childhood but also for a healthy later life.

Family is a basic unit of society that children encounter in their life, which encourages interaction between family members and inevitably gives an impact on one another [4]. Especially family is a proximal food environment and has an impact on food choices, dietary behaviors, and food intake of children through the mechanisms such as parent role modeling, social support, and social norms [5]. Thus, family is considered to be an important factor associated with dietary intake in children.

In recent decades, family structure has changed with an increase in divorce, remarriage, non-marital childbearing, and dual earner families in Korean society: there is an increase in custodial grandparent or single parent families while decrease in traditional nuclear family consisting of parents and unmarried sons and daughters [6]. A large volume of studies supported that there were association between the family structure or household type and children's eating habits and health status. Some studies indicated that children in single parent family, reconstituted families, or family without patents were more likely to have unhealthy eating habits such as irregular breakfast consumption, eating less vegetable, and drinking alcohol compared to children living with both parents [7-10]. Also, there are several studies which suggest a positive association between child health including obesity, asthma and family structure in single-mother families and custodial grandparent families [11-17].

While several studies have investigated an association of family structure with children's eating habit, health and well-being [7-17], studies that have examined the association with children's food intake are scarce. Diversity and adequacy of foods which compose meals should also be taken into account in assessment of nutritional status [18]. Therefore, this

\footnotetext{
${ }^{\S}$ Corresponding Author: Jae Eun Shim, Tel. 82-42-280-2469, Fax. 042-280-2468, Email. jshim@dju.kr

Received: December 12, 2013, Revised: April 21, 2014, Accepted: April 27, 2014

This is an Open Access article distributed under the terms of the Creative Commons Attribution Non-Commercial License (http://creativecommons.org/licenses/by-nc/3.0/) which permits unrestricted non-commercial use, distribution, and reproduction in any medium, provided the original work is properly cited.
} 
study aims to examine the association between family structure and food group intake of children aged 3-18 years in Korea.

\section{SUBJECTS AND METHODS}

\section{Study subject}

The analyses were performed using data from the Fifth Korea National Health and Nutrition Examination Survey (KNHANES V) 2010-2011, which was conducted by Korea Centers for Disease Control and Prevention (KCDC) [19]. The survey participants were recruited from a nationally representative sample of non-institutionalized Korean population aged 1 year or more. The survey consisted of Health Interview Survey, Health Examination Survey, and Nutrition Survey and a rolling survey sampling design and a complex, stratified multistage, probabilitycluster sampling design were applied.

This study focused on 3,663 children aged 3-18 years (21\% of a total of 17,476 KNHANES V (2010-2011) participants). The analytic data set included data from a total of 3,217(1,678 boys and 1,539 girls) children with complete data on variables for household information, dietary intake by using single 24-hour recall method and sampling weights (Fig. 1). Children's family structure was classified into three groups: 'Living with both parents', 'Living with one parent', and 'Living without parents'. The distribution of children by family structure showed that $6.6 \%$ lived without parents, $12.3 \%$ lived with one parent, and $81.1 \%$ lived with both parents.

\section{Definition of variables}

Living area was classified into three groups: 'Metropolises', 'Small and medium-sized cities', and 'Rural' areas. Because of small proportion of some household income quartile (living with both parents: Q1 7.4\%, living without parents: Q3 7.5\%, Q4 7.6\%), quartile classification of household income in KNHANES data had coalesced into two groups: 'Below $50^{\text {th }}$ percentile' and

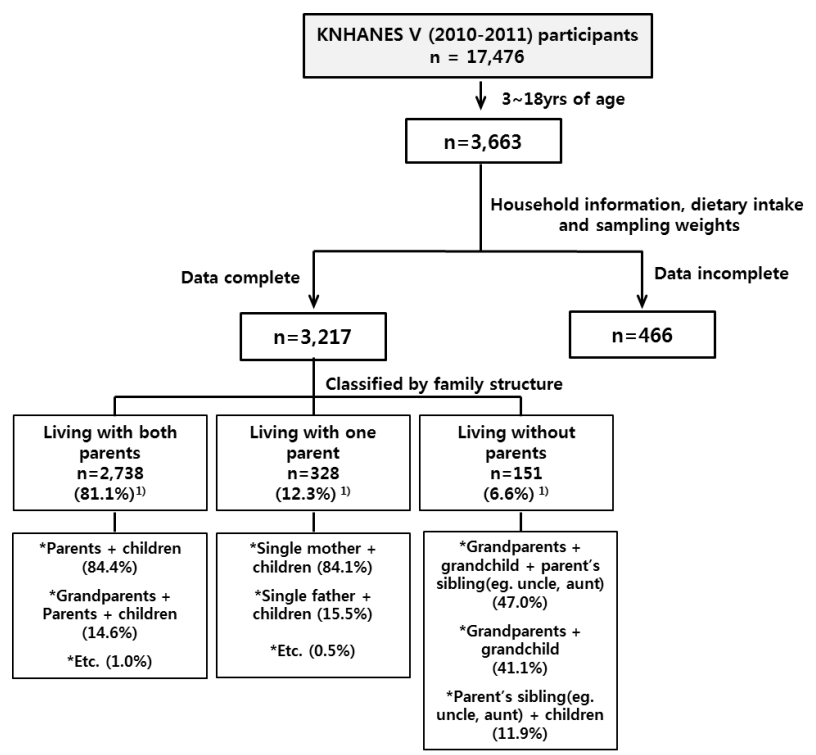

Fig. 1. Flow chart of subject disposition. ${ }^{1)}$ Sample weights for health interview survey were applied for estimates
'Over $50^{\text {th }}$ percentile'.

Dietary intakes of subjects were obtained using 24-hour recall data. Consumed foods were categorized into five major food groups ('Grains', 'Meat, Fish, Eggs, and Beans', 'Vegetables', 'Fruits', and 'Milk and Dairy products') and the amount was converted into number of servings based on the Korean Food Guidance System (KFGS) [20]. Food items unrepresented in KFGS and mixed food items (e.g. hamburger, dumpling) were classified into five food groups and the amount was converted into servings based on newly-developed food group database established by Song et al. [21] In the food group database, the other foods except representatives in KFGS and mixed dishes were classified on the basis of energy, nutrient contents, and recipe. Juice and ice cream were excluded from 'Fruits' and 'Milk and Dairy products' groups respectively in this study.

To evaluate children's food group intake, individual food group score was calculated by using percent adherence to the recommended servings in the KFGS [20]. The KFGS, which is applicable to individuals aged 3 and more years, provides recommended number of servings for each food group according to age and sex. In this study, children's intake of 'Grains', 'Meat, Fish, Eggs, and Beans', 'Vegetables', 'Fruits', and 'Milk and Dairy products' was evaluated.

Individual food group score was calculated as follows:

Food group score $=$ (number of servings consumed $\div$ number of servings recommended in KFGS) x 100 .

Maximum score was 100 for any food group and if intake was higher than $100 \%$ of recommended servings, a score of 100 was assigned. Scores below 100 indicates that the food group was consumed less than the KFGS recommendation, and score equal to 100 implies that the food group intake is equivalent to or more than the KFGS recommendation. 'Food group mean score' was obtained from the average of five individual scores.

\section{Statistical analyses}

Analyses were performed using SAS 9.3 software (Statistic Analysis System; version 9.3, SAS Institute, Cary, NC, USA.). Taylor series method was applied to account for multiple stage sampling. All estimates were produced to represent Korean population using the sample weight for joint analysis of nutrition survey and health interview survey. Descriptive statistics were presented as percentage frequency or mean and standard error. Children's characteristics by family structure were analyzed with Rao-Scott Chi-Square test and Analysis of Variance test (ANOVA). Multivariate analysis using Analysis of Covariance test (ANCOVA) was applied to control for age, sex, number of family members, and household income. The differences in mean values of food group scores of participants with different family structure were analyzed. To examine the association between family structure and food group scores of children, multivariate regression model was tested. In the test, five independent variables were included: family structure, age, sex, number of family members and household income. Categorical variables such as family structure, sex, and household income were included in the model as dummy variables. Statistical significance was set at $a=0.05$. 


\section{RESULT}

\section{Subject characteristics}

Characteristics of the children by family structure are presented in Table 1. Lower percentage of young children (3-5 yrs of age) and higher percentage of older children (12-18 yrs of age) lived with one parent $(P<0.001)$. Majority of children living without parents (84.9\%) or living with one parent $(71.0 \%)$ were distributed below the 50th percentile of household income. Number of family members was significantly different among children with different family structure.

\section{Food group scores}

Children's food group scores and mean scores of individual food group scores were presented in Table 2. Results showed that mean scores of total subjects were less than 50 for both 'Fruits' and 'Milk and Dairy products', whereas the mean was approximately 90 for 'Grains'. After adjusting for age, sex, number of family members, and household income, children living with both parents had higher scores in 'Fruits' $(P<0.01)$ and 'Milk and Dairy products' $(P<0.05)$ compared to children living with one parent. The mean scores of individual food group scores also appeared to be higher in children living with both parents than those with one parent $(P<0.001)$, while 'Grains', 'Meat, Fish, Eggs, and Beans', and 'Vegetables' scores of children with different family structure were similar to each other.

Association between family structure and children's food group scores

Regression models for food group scores and mean score of individual food group scores with five independent variables that consists of children's family structure and socio-demographic factors were applied (Table 3). The selected models were statistically significant except those for 'Meat, Fish Eggs, and Beans' group score. Socio-demographic factors associated with

Table 1. Children's characteristics by family structure ${ }^{1)}(n=3,217)$

\begin{tabular}{|c|c|c|c|c|c|}
\hline \multirow{2}{*}{ Variables } & Living with both parents & Living with one parent & Living without parents & Total & \multirow{2}{*}{$P^{2)}$} \\
\hline & $\%$ & $\%$ & $\%$ & $\%$ & \\
\hline \multicolumn{6}{|l|}{ Sex } \\
\hline Male & 52.6 & 52.1 & 53.6 & 52.6 & \multirow{2}{*}{0.071} \\
\hline Female & 47.4 & 47.9 & 46.4 & 47.4 & \\
\hline \multicolumn{6}{|l|}{ Age group } \\
\hline 3-5 yrs & 15.6 & 7.5 & 14.4 & 14.5 & \multirow{3}{*}{$<.001$} \\
\hline 6-11 yrs & 39.1 & 25.2 & 34.8 & 37.1 & \\
\hline $12-18$ yrs & 45.3 & 67.3 & 50.8 & 48.3 & \\
\hline \multicolumn{6}{|l|}{ Living area } \\
\hline Metropolises & 42.1 & 53.2 & 40.0 & 43.3 & \multirow{3}{*}{0.266} \\
\hline Small and medium-sized cities & 39.6 & 33.7 & 40.8 & 38.9 & \\
\hline Rural areas & 18.4 & 13.1 & 19.2 & 17.8 & \\
\hline \multicolumn{6}{|l|}{ Household income ${ }^{4)}$} \\
\hline Below $50^{\text {th }}$ percentile & 39.5 & 71.0 & 84.9 & 46.3 & \multirow{2}{*}{$<.001$} \\
\hline Over $50^{\text {th }}$ percentile & 60.5 & 29.0 & 15.1 & 53.7 & \\
\hline \multicolumn{6}{|l|}{ No. of family members } \\
\hline & $4.4 \pm 0.03^{3) \mathrm{a}}$ & $3.6 \pm 0.1^{b}$ & $4.0 \pm 0.2^{\mathrm{ab}}$ & $4.3 \pm 0.03$ & $<.001$ \\
\hline
\end{tabular}

1) Sample weights for health interview survey were applied for estimates.

2) Rao-Scott $x^{2}$ test and ANOVA with Tukey's test.

${ }^{3)}$ Data are expressed as mean \pm SE. Values in a row with different letters are significantly different at $P<0.05$.

4) Missing $=35$

Table 2. Children's food group score by family structure ${ }^{1)}(n=3,182)$

\begin{tabular}{|c|c|c|c|c|c|}
\hline \multirow{2}{*}{ Variables } & Living with both parents & Living with one parent & Living without parents & Total & \multirow{2}{*}{$P^{2)}$} \\
\hline & mean $\pm \mathrm{SE}$ & mean $\pm \mathrm{SE}$ & mean $\pm \mathrm{SE}$ & mean $\pm \mathrm{SE}$ & \\
\hline \multicolumn{6}{|l|}{ Food group score } \\
\hline Grains & $86.7 \pm 0.5$ & $85.1 \pm 1.7$ & $84.5 \pm 2.7$ & $86.4 \pm 0.5$ & 0.489 \\
\hline Meat, Fish, Eggs, and Beans & $71.0 \pm 0.8$ & $67.9 \pm 2.7$ & $66.6 \pm 3.2$ & $70.3 \pm 0.8$ & 0.233 \\
\hline Vegetables & $67.0 \pm 0.9$ & $63.5 \pm 2.8$ & $68.6 \pm 2.9$ & $66.6 \pm 0.8$ & 0.449 \\
\hline Fruits ${ }^{3)}$ & $44.6 \pm 1.4^{\mathrm{a}}$ & $34.6 \pm 2.4^{b}$ & $38.3 \pm 4.0^{\mathrm{ab}}$ & $42.9 \pm 1.2$ & 0.001 \\
\hline Milk and Dairy ${ }^{4)}$ & $45.2 \pm 1.0^{\mathrm{a}}$ & $38.3 \pm 2.6^{b}$ & $40.0 \pm 3.5^{\mathrm{ab}}$ & $44.0 \pm 0.9$ & 0.029 \\
\hline Food group mean score & $62.9 \pm 0.4^{\mathrm{a}}$ & $57.9 \pm 1.5^{\mathrm{b}}$ & $59.6 \pm 1.9^{\mathrm{ab}}$ & $62.0 \pm 0.4$ & $<.001$ \\
\hline
\end{tabular}

1) Sample weights for joint analysis of nutrition survey and health interview survey were applied for all estimates.

2) ANCOVA adjusted for age, sex, number of family members and household income with Tukey's test. Values in a row with different letters are significantly different at $P$ $<0.05$.

3) Juice excluded.

4) Ice cream excluded. 
Table 3. Regression model of food group scores ${ }^{1)}(n=3,182)$

\begin{tabular}{|c|c|c|c|c|c|c|c|c|c|c|c|c|}
\hline \multirow{2}{*}{ Independent variables } & \multicolumn{2}{|c|}{ Grains } & \multicolumn{2}{|c|}{$\begin{array}{c}\text { Meat, Fish, Eggs, and } \\
\text { Beans }\end{array}$} & \multicolumn{2}{|c|}{ Vegetables } & \multicolumn{2}{|c|}{ Fruits ${ }^{3)}$} & \multicolumn{2}{|c|}{ Milk and Dairy ${ }^{4)}$} & \multicolumn{2}{|c|}{$\begin{array}{l}\text { Food group mean } \\
\text { score }\end{array}$} \\
\hline & $\beta \pm S E$ & $P$ & $\beta \pm S E$ & $P$ & $\beta \pm S E$ & $P$ & $\beta \pm S E$ & $P$ & $\beta \pm S E$ & $P$ & $\beta \pm S E$ & $P$ \\
\hline \multicolumn{13}{|l|}{ Family structure ${ }^{2)}$} \\
\hline Living without parents & $-2.2 \pm 2.8$ & 0.423 & $-4.4 \pm 3.3$ & 0.187 & $1.6 \pm 3.0$ & 0.588 & $-6.3 \pm 4.2$ & 0.129 & $-5.1 \pm 3.6$ & 0.159 & $-5.1 \pm 3.6$ & 0.090 \\
\hline Living with one parent & $-1.5 \pm 1.7$ & 0.369 & $-3.1 \pm 2.9$ & 0.292 & $-3.5 \pm 3.0$ & 0.245 & $-10.1 \pm 2.8$ & $<0.001$ & $-6.9 \pm 2.9$ & 0.016 & $-6.9 \pm 2.9$ & 0.001 \\
\hline
\end{tabular}

the food intake of children were different in each food group. Family structure was associated with 'Fruits', 'Milk and Dairy products' scores and 'Food group mean score,' after adjusting for age, sex, number of family members, and household income. Household income was also strongly associated with food group scores such as 'Grains', 'Milk and Dairy' groups and 'Food group mean score'.

\section{DISCUSSION}

This study was to examine the association between family structure and food group intake of children in Korea. The study results represented that children living with both parents had higher food group scores for 'Fruits', 'Milk and Dairy products', and the mean score of individual food group scores compared to children living with one parent. Individual food group scores were associated with different socio-demographic factors of children. Food groups of which consumption was associated with family structure of children were 'Fruits', 'Milk and Dairy products' and overall intake of the five food groups.

In this study, about $12.3 \%$ of children were lived with one parent. It was similar to the results of the 2010 Population and Housing Census [6], while the proportion was lower than those of foreign countries $[7,8,10,14]$. Other domestic studies on elementary school children from low-income households [22,23] showed that higher proportion of children lived with one parent than current study but the results were not produced from representative samples.

In this study, children had insufficient intakes of both 'Fruits' and 'Milk and Dairy products' whatever the family structure is. This result was consistent with a report of Shim et al. [24] that had used the 2001 KNHANES data to examine the dietary intake of children aged 6-18 years by household income level. Low intake of 'Fruits' and 'Milk and Dairy products' groups is common dietary intake problem in children, which can ultimately lead to health problems related to vitamin C, calcium, and riboflavin deficiency. Sufficient intake of milk and dairy products as important souses of calcium is necessary to support growth during childhood. Furthermore, adequate calcium supply to achieve higher peak bone mass is crucial during this growth period in order to maintain high bone density in later life [25]. Therefore, more rigorous approaches are needed for nutrition education and policy that aim to promote the intake of 'Fruits' and 'Milk and Dairy products' for children.

Children living with both parents had higher scores for 'Fruits' and 'Milk and Dairy products' groups and overall food groups compared to children living with one parent. A study [26] on childcare expenses per children indicated that grocery expenses in single-headed households was $89.2 \%$ of the expenses in total household and the expenses in beneficiaries of basic livelihood assistance program was $72.1 \%$. Healthy foods such as fresh vegetables, fruits, and milk and dairy products were more expensive on a per calorie basis than less healthy foods (e.g. fat, sugar, refined grains) and low income households tended to spend and consume less fruits than high income households $[27,28]$. Therefore, it can be assumed that single-headed households, who also happen to have low household income, have a tendency to choose foods that meet their energy needs at lower cost rather than to purchase foods of greater variety and adequacy at higher cost.

The lower food group scores in single-headed households are thought to be partially due to lack of care including preparation of meals composed of diverse and adequate amount of foods. A large volume of literature suggests that family meals are associated with higher fruit and vegetable intake [29-32]. A qualitative study of perspectives on healthy family meals from single-headed and dual-headed households identified several reasons for barriers to family meals [33]. Parents from dualheaded households reported that busy schedules were major barriers to having family meal. While parents from singleheaded households identified cost was a major barrier to health family meal, they also reported that they felt burden of sole responsibility to carry out family meal.

Despite the fact that children living without parents had the lowest household income, intake of food groups and 'mean food group score' were not statistically different when compared with the children living with parents. An explanation for this observation is that even if children are not living with their parents, their dietary intake may not be compromised especially when they are under direct care from their grandparents or other household members. As shown in Fig. 1, household of 'children living without parents' consisted of couple of adults.

Furthermore, a study by Sung et al. [34] suggested that interpersonal relationship in children was similar or slightly better in children under custodial care from grandparents than those living with their parents. Children with custodial grandparents had much more positive aspects in quality of life, and criminal behaviors were 2-3 folds lower than children in single-parent family. As such, it can be understood that grandparents or other care-giving household members might give 
positive influence on children.

There are several limitations in this study. First, this study used data from KNHANES V (2010-2011) with one-day dietary intake. Second, this study mainly focused on the influence of family structure on food group intake of children. However factors such as siblings living in the household, availability and accessibility of food items within home, neighborhood food environments such as restaurants, food stores, and media could influence on food choice and intake in children but were not accounted for [5]. Current study classified children's family structure into three groups ('Living with both parents', 'Living with one parent', and 'Living without parents'). In reality, family structures are a lot more complicated. Thus, more detailed classification system could be required (e.g. reconstituted family such as mother-stepfather, father-stepmother; one parent family such as non-marital childbearing, married but divorce or bereavement; custodial grandparent family). Third, current study was based on secondary data and potential confounding variables could not be fully controlled (e.g. primary care giver's socio-demographic characteristics). Nevertheless, this is the first study which evaluate children's food group intake in reference to 2010 revised KFGS by family structure, and identify the relationship between children's family structure and their food group intake. This study documented socio-demographic characteristics related to food intake of children, which could be a basis for national policy, project, and interventions in regard to children's family structure aiming to solve the nutrition problems of children in Korea.

\section{REFERENCE}

1. Brown JE, Isaacs JS. Child and preadolescent nutrition. In: Brown $J E$, Isaacs JS, editors. Nutrition through the Life Cycle. 4th ed. Belmont (CA): CENGAGE Learning; 2010. p.311.

2. Boyle MA, Holben DH. Community Nutrition in Action: An Entrepreneurial Approach. 5th ed. Belmont (CA): CENGAGE Learning; 2009.

3. Serdula MK, Ivery D, Coates R, Freedman DS, Williamson DF, Byers T. Do obese children become obese adults? A review of the literature. Prev Med 1993;22:167-77.

4. Lee GH. Human Behavior and the Social Environment. Seoul: Gong Dong Che; 2006.

5. Story M, Kaphingst KM, Robinson-O'Brien R, Glanz K. Creating healthy food and eating environments: policy and environmental approaches. Annu Rev Public Health 2008;29:253-72.

6. Statistics Korea. Population and Housing Census. Daejeon: Statistics Korea; 2010.

7. Jørgensen A, Pedersen TP, Meilstrup CR, Rasmussen M. The influence of family structure on breakfast habits among adolescents. Dan Med Bull 2011;58:A4262.

8. Levin KA, Kirby J. Irregular breakfast consumption in adolescence and the family environment: underlying causes by family structure. Appetite 2012;59:63-70.

9. Levin KA, Kirby J, Currie C. Adolescent risk behaviours and mealtime routines: does family meal frequency alter the association between family structure and risk behaviour? Health Educ Res 2012;27:24-35.

10. Stewart SD, Menning CL. Family structure, nonresident father involvement, and adolescent eating patterns. J Adolesc Health
2009:45:193-201.

11. Chen AY, Escarce JJ. Family structure and childhood obesity, Early Childhood Longitudinal Study - Kindergarten Cohort. Prev Chronic Dis 2010;7:A50.

12. Schmeer KK. Family structure and obesity in early childhood. Soc Sci Res 2012;41:820-32.

13. Formisano A, Hunsberger $M$, Bammann $K$, Vanaelst $B$, Molnar $D$, Moreno LA, Tornaritis M, Veidebaum T, Lissner L, Barba G, Siani A. Family structure and childhood obesity: results of the IDEFICS Project. Public Health Nutr 2013:1-9.

14. Dawson DA. Family structure and children's health and well-being: data from the 1988 National Health Interview Survey on Child Health. J Marriage Fam 1991;53:573-84.

15. Montgomery LE, Kiely JL, Pappas G. The effects of poverty, race, and family structure on US children's health: data from the NHIS, 1978 through 1980 and 1989 through 1991. Am J Public Health 1996;86:1401-5.

16. Thompson SJ, Auslander WF, White NH. Comparison of singlemother and two-parent families on metabolic control of children with diabetes. Diabetes Care 2001;24:234-8.

17. Bronte-Tinkew J, DeJong G. Children's nutrition in Jamaica: do household structure and household economic resources matter? Soc Sci Med 2004;58:499-514.

18. Jeong H, Kim BH, Song YJ, Lee JE. Nutrition in Public Health. Seoul: Yang Seo Won; 2013.

19. Ministry of Health and Welfare, Korea Centers for Disease Control and Prevention. Korea Health Statistics 2010: Korea National Health and Nutrition Examination Survey (KNHANES V-1). Cheongwon: Korea Centers for Disease Control and Prevention; 2011.

20. The Korean Nutrition Society. Dietary Reference Intakes for Koreans. Seoul: The Korean Nutrition Society; 2010.

21. Song SJ, Jung HJ, Shim JE, Paik HY. Assessment of food group intake in Korean population with a newly-developed food group database. J Food Compost Anal. Forthcoming 2014.

22. Nam KH, Kim YM, Lee GE, Lee YN, Joung H. Physical development and dietary behaviors of children in low-income families of Seoul area. Korean J Community Nutr 2006;11:172-9.

23. Lee $\mathrm{JH}$, Son EY, Kim Y, Lim Y. Dietary habits and nutrition knowledge of children using community food service centers according to family type. Korean J Nutr 2011;44:255-63.

24. Shim JE, Yoon J, Lee K, Kwon S. Evaluation of dietary intake of Korean school-aged children from low-income families by comparing with the Korean food guide: analysis of the data from the 2001 National Health and Nutrition Survey. Korean J Nutr 2009;42: 691-701.

25. Nicklas TA. Calcium intake trends and health consequences from childhood through adulthood. J Am Coll Nutr 2003;22:340-56.

26. Kim YW. Family type and childcare expenses. Health Welf Policy Forum 2013:57-68.

27. Blisard N, Stewart H, Jolliffe D. Low-Income Households' Expenditures on Fruits and Vegetables: United States Department of Agriculture, Agricultural Economic Report No.833. Washington, D.C.: United States Department of Agriculture; 2004.

28. Drewnowski A, Specter SE. Poverty and obesity: the role of energy density and energy costs. Am J Clin Nutr 2004;79:6-16.

29. Gable S, Lutz S. Household, parent, and child contributions to childhood obesity. Fam Relat 2000;49:293-300.

30. Gillman MW, Rifas-Shiman SL, Frazier AL, Rockett HR, Camargo CA 
Jr, Field AE, Berkey CS, Colditz GA. Family dinner and diet quality among older children and adolescents. Arch Fam Med 2000;9: 235-40.

31. Neumark-Sztainer D, Hannan PJ, Story M, Croll J, Perry C. Family meal patterns: associations with sociodemographic characteristics and improved dietary intake among adolescents. J Am Diet Assoc 2003;103:317-22.

32. Cason KL. Family mealtimes: more than just eating together. J Am
Diet Assoc 2006;106:532-3.

33. Berge JM, Hoppmann C, Hanson C, Neumark-Sztainer D. Perspectives about family meals from single-headed and dual-headed households: a qualitative analysis. J Acad Nutr Diet 2013;113:1632-9.

34. Sung YS, Kim YH, Song UG. Analysis of Children.Youth Status by Family Type and Research on Countermeasures - Focused on Children-Youth in Family with Parents. Seoul: National Youth Policy Institute; 2011. 